\title{
First Geodetic Observations Using New VLBI Stations ASKAP-29 and WARK12M
}

\author{
Leonid Petrov $^{\mathrm{A}, \mathrm{G}}$, Chris Phillips ${ }^{\mathrm{B}}$, Tasso Tzioumis ${ }^{\mathrm{B}}$, Bruce Stansby ${ }^{\mathrm{C}}$, Cormac Reynolds ${ }^{\mathrm{C}}$, \\ Hayley E. Bignall ${ }^{\mathrm{C}}$, Sergei Gulyaev ${ }^{\mathrm{D}}$, Tim Natusch ${ }^{\mathrm{D}}$, Neville Palmer ${ }^{\mathrm{E}}$, David Collett ${ }^{\mathrm{F}}$, \\ John E. Reynolds ${ }^{\mathrm{B}}$, Shaun W. Amy ${ }^{\mathrm{B}}$, Randall Wayth ${ }^{\mathrm{C}}$, and Steven J. Tingay ${ }^{\mathrm{C}}$ \\ A ADNET Systems, Inc./NASA GSFC, Code 610.2, Greenbelt, MD 20771, USA \\ ${ }^{B}$ CSIRO Astronomy and Space Science, PO Box 76, Epping, NSW 1710, Australia \\ ${ }^{\mathrm{C}}$ International Centre for Radio Astronomy Research, Curtin University, Bentley, \\ WA 6102, Australia \\ D Institute for Radio Astronomy and Space Research, Auckland University of Technology, \\ Private Bag 92006, Auckland 1142, New Zealand \\ ${ }^{\mathrm{E}}$ GNS Science, PO Box 30368, Lower Hutt 5040, New Zealand \\ F Land Information New Zealand, 160 Lambton Quay, PO Box 5501, \\ Wellington 6145, New Zealand \\ ${ }^{\mathrm{G}}$ Corresponding author. Email: Leonid.Petrov@1petrov.net
}

Received 2010 December 20, accepted 2011 February 9

\begin{abstract}
We report the results of a successful 7-hour 1.4 GHz Very Long Baseline Interferometry (VLBI) experiment using two new stations, ASKAP-29 located in Western Australia and WARK12M located on the North Island of New Zealand. This was the first geodetic VLBI observing session with the participation of these new stations. We have determined the positions of ASKAP-29 and WARK12M. Random errors on position estimates are 150-200 $\mathrm{mm}$ for the vertical component and 40-50 $\mathrm{mm}$ for the horizontal component. Systematic errors caused by the unmodeled ionosphere path delay may reach $1.3 \mathrm{~m}$ for the vertical component.
\end{abstract}

Keywords: instrumentation: interferometers — techniques: interferometric — reference systems

\section{Introduction}

As part of the joint bid by Australia and New Zealand to host the multi-billion dollar Square Kilometre Array (SKA), both countries are investing heavily in advanced technologies for radio astronomy. In Australia this follows a strong tradition in radio astronomy and is expressed in the construction of the Australian SKA Pathfinder (ASKAP) on the western edge of the Australian continent (Johnston et al. 2008). Radio astronomy in New Zealand has links stretching back to the work of Elizabeth Alexander on solar radio emission (Alexander 1946). John Bolton and Gordon Stanley used a cliff interferometer to obtain rising and setting records of various radio sources (Bolton 1982); measurements made near Sydney and Auckland allowed them to identify mysterious 'radiostars' with well-known supernova remnants and galaxies (Bolton, Stanley \& Slee 1949). The first successful Very Long Baseline Interferometry (VLBI) experiment between Australia and New Zealand was made in 2005 with a 6-m radio telescope near Auckland (Karaka) and the Australia Telescope Compact Array (ATCA) (Gulyaev et al. 2005; Tingay et al. 2006). In 2008, Auckland University of Technology commissioned a new 12-m antenna at Warkworth near Auckland New Zealand's first research-capable radio telescope (Gulyaev \& Natusch 2009).

A joint Australia and New Zealand collaborative project is focused on developing both the ASKAP and Warkworth facilities as part of regional and global VLBI arrays, for the purposes of both astronomy and geodesy. Due to the dearth of land mass in the Southern Hemisphere, both astronomical and geodetic VLBI has suffered in the past, with the radio telescopes used for VLBI restricted to the south-east 'corner' of the Australian continent, one telescope in South Africa and occasional use of radio telescopes in Antarctica and South America.

The additional capability gained by adding radio telescopes located in Western Australia and New Zealand is substantial, increasing the angular resolution of the Australasian array by a factor of approximately four, admitting a range of astronomy science goals described in Johnston et al. (2008).

High-precision astrometry is likely to be an important part of the science case for the high-angular resolution component of the SKA, in particular for precise determination of the distances of radio pulsars in the galaxy, to be 
used in various tests of fundamental physics (Smits et al. 2010). In order to achieve the astrometric performance required, the SKA will need to undertake astrometry utilising distributed clusters of small antennas operating as phased arrays. This is a departure from standard precision astrometry and our work using ASKAP as part of a VLBI array for astrometry will be an important testbed for the demonstration of these techniques.

The $12-\mathrm{m}$ radio telescope WARK I $2 \mathrm{M}^{1}$ is intended to be used as a part of the Australian Long Baseline Array (LBA), for spacecraft monitoring, and for VLBI observations in the framework of the International VLBI Service for Geodesy \& Astrometry (IVS) ${ }^{2}$ and the AuScope project. $^{3}$

ASKAP-29 is an element of the ASKAP array of 36 identical dishes using Phased Array Feed (PAF) technologies, expected to be fully operational by 2013. ASKAP will undertake very wide field survey science in continuum and spectral line modes and is also intended to be used as a part of regional and global VLBI networks for a variety of projects. $^{4}$

As a part of commissioning the new antennas, the positions of antenna reference points should be determined. A reference point is defined as the point of the projection of the movable elevation axis onto the fixed azimuthal axis. For the analysis of VLBI source-imaging experiments made in a phase-referencing mode, the projection of a baseline vector (i.e. vector between antenna reference points) onto the tangential image plane should be known with errors not exceeding tens of centimetres, otherwise the image will be smeared (Charlot 2002). For astrometry applications, angular position accuracies of tens of $\mu$ as are required, making the requirements on the accuracy of station positions much more stringent: $5-10 \mathrm{~mm}$. One way of estimating the position of the antenna reference points is through analysis of a combination of a ground survey of markers attached to the antennas from a local network around the station and GPS observations from the points at the local network (see e.g. Sarti, Sillard \& Vittuari 2004; Sarti, Abbondanza \& Vittuari 2009). Another way to estimate station positions is to use the VLBI technique itself to determine group delays and then derive reference point positions from these group delay measurements. The advantage of this approach is that it also provides useful diagnostics on the VLBI equipment

The first fringe-test experiment between ASKAP-29 and WARKI2M was made in April 2010. First fringes on baselines between ASKAP-29 and MOPRA were found on 2010 April 22 and on baselines to WARK I $2 \mathrm{M}$ on the following day. This success prompted three first science

\footnotetext{
${ }^{1}$ WARKI2M and ASKAP-29 are identifiers for specific VLBI antennas: near Warkworth, New Zealand and at the Murchison Radio-Astronomy Observatory, Western Australia, respectively.

${ }^{2}$ http: / / ivscc.gsfc.nasa.gov/.

${ }^{3}$ http: / / www . auscope.org.au/.

${ }^{4}$ http: //www.atnf.csiro.au/SKA/.
}

experiments using the full LBA network with the participation of the two new stations: (1) imaging observations of PKS 1934-638 on 2010 April 29-30 (Tzioumis et al. 2010a), (2) a geodetic experiment on 2010 May 07, and (3) imaging onbservations of Cen-A on 2010 May 09 (Tingay et al. 2010, in preparation).

We report here results from the geodetic experiment that was conducted at ASKAP-29 and during the first geodetic observing session on 2010 May 07. The goal of this experiment was to determine the position of the antennas with decimeter accuracy and to collect diagnostic data. The characteristics of new antennas are presented in section 2. The experiment and its analysis are described in sections 3 and 4. Concluding remarks are made in section 5 .

\section{New Antennas}

\subsection{ASKAP-29}

The Australian SKA Pathfinder (ASKAP) is an advancedtechnology radio telescope currently under construction at the Murchison Radio-astronomy Observatory (MRO) in Western Australia. The ASKAP array comprises 36 fully steerable dish antennas of 12-metre aperture fitted with phased array feed (PAF) receivers, giving an instantaneous field of view of approximately 30 square degrees with an instantaneous bandwidth of $300 \mathrm{MHz}$ in the range $700-1800 \mathrm{MHz}$.

The first antenna, ASKAP-29, was commissioned in March 2010 and was temporarily fitted with a conventional horn (single-pixel) L-band feed $(1.4 \mathrm{GHz})$ and uncooled receiver. Five additional antennas were completed in Q4 2010 with the balance to be commissioned throughout 2011.

The antenna is of a prime focus design with an unshaped paraboloidal reflector of $\mathrm{f} / \mathrm{D}$ 0.5. The original specifications on surface accuracy were $1 \mathrm{~mm}$ root mean square (rms). However, measurements showed that the surface accuracy of the ASKAP-29 reflector and six other identical antennas manufactured by November 2010 is better than $0.5 \mathrm{~mm}$ rms over the full elevation range. There is some flexure in the position of the focus at a level of few mm over the full elevation range, but this can be compensated for if required. This level of surface accuracy implies that the antenna will be usable at frequencies as high as $\mathrm{Ka}$ band $(30 \mathrm{GHz})$ and possibly at $\mathrm{Q}$ band (43 GHz). A quadripod supports the prime focus platform with maximum payload of about $200 \mathrm{~kg}$. Additional bracing supports on each leg have been added to the original design to reduce flexure.

An unusual feature of the antenna is the three-axis mount. Above the familiar azimuth and elevation axes is the so-called polarization axis, allowing the entire reflector to be rotated about its optical axis. This design was specified to allow full parallactic angle tracking for high dynamic range in imaging observations, as it improves modeling of far-out sidelobes caused by the quadripod. An equatorial ('HA-Dec') design naturally maintains a 


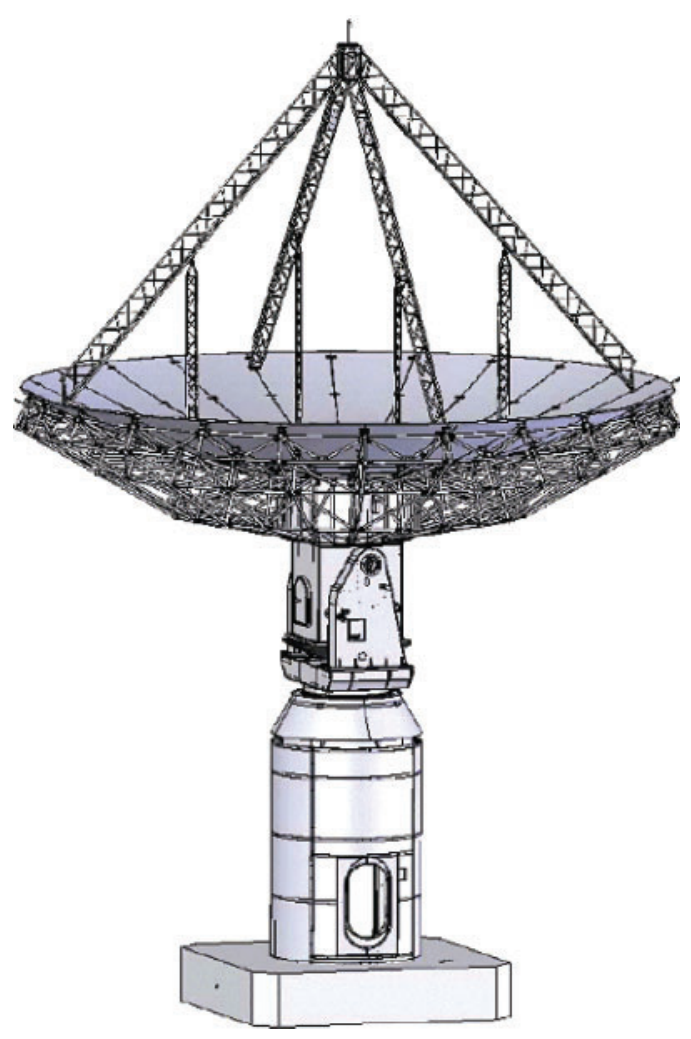

Figure 1 ASKAP antenna.

constant orientation of its focal plane with respect to the celestial sphere when tracking a fixed point in celestial coordinates. However this type of mount usually suffers restricted sky coverage due to limited rotation in hour angle.

Another feature of the antenna is the relatively large pedestal specified to accommodate the analogue and digital electronics for the PAF receiver with its 188 individual ports. The 54th Research Institute of China Electronics Technology Group Corporation (known as CETC54) are contracted to design, manufacture and install all thirty-six antennas at the MRO.

The baseband data at ASKAP-29 were recorded directly to disk utilising a custom recording system developed at Curtin University. The recording systems were assembled from consumer off-the-shelf components. The recorders were interfaced with the CSIRO supplied analogue downconverters. The systems recorded the data to disk at the native sampler/digitizer sample size of 16 bits.

The 16-bit sample size gives an aggregate data rate when sampled at the Nyquist-Shannon rate over 2 polarization channels of $4 \mathrm{Gbit} / \mathrm{s}$. The recorder system comprised a Signatec PX14400 PCIe sampler/digitizer mounted in a server-class computer. The systems were built in a 16-disk chassis populated with SATA hard drives. The recording system utilised a Linux software RAID, and the 16 disks where subdivided into 8-disk RAID units. The recorded data were converted to the LBA format with 2-bit samples after the experiment using software employing automatic gain control in the

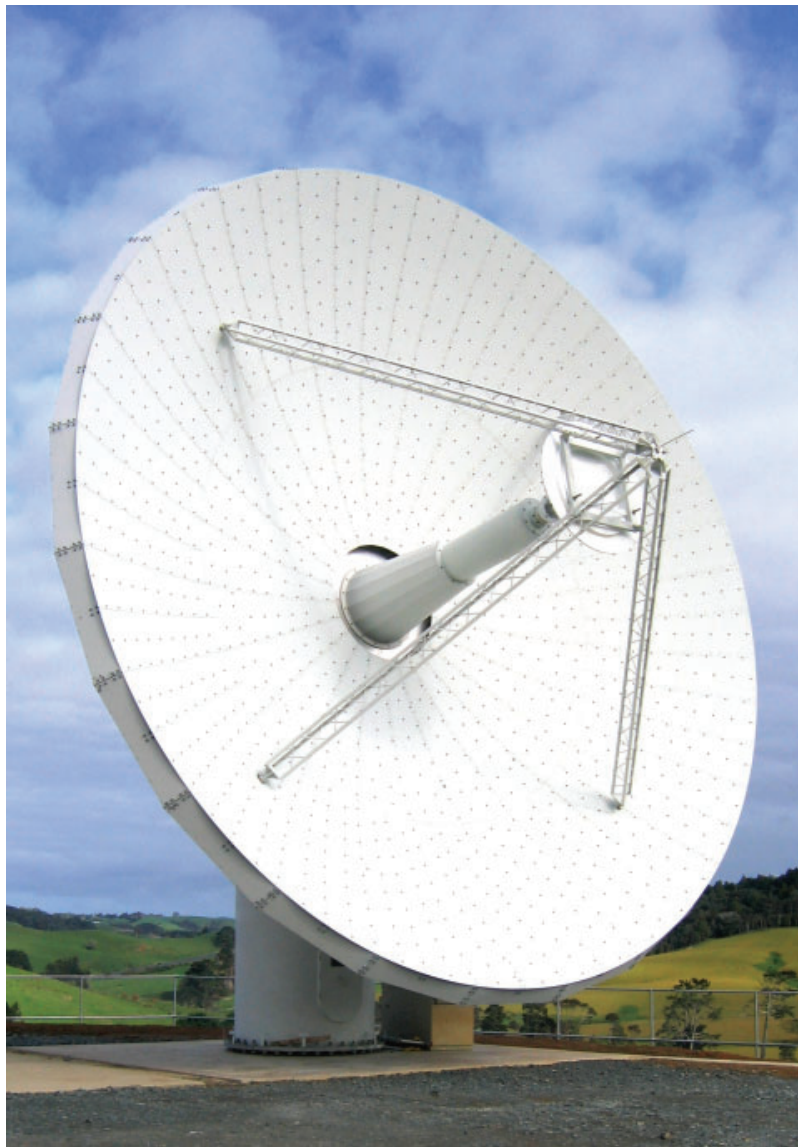

Figure 2 WARKI $2 \mathrm{M}$ antenna.

conversion process in order to match the configuration of LBADR recorders used at other stations.

\subsection{WARK12M}

The New Zealand 12-m radio telescope (see Figure 2) is located some $60 \mathrm{~km}$ north of the city of Auckland, near the township of Warkworth. It was manufactured by Patriot Antenna Systems (now Cobham Antenna Systems), USA. The antenna specifications are provided in Table 2. The radio telescope was originally designed to operate at $\mathrm{S}$ $(2.3 \mathrm{GHz})$ and $\mathrm{X}$ bands $(8.4 \mathrm{GHz})$ and it was supplied with an $\mathrm{S} / \mathrm{X}$ dual-band dual-polarisation feed. Photogrammetry observations showed that the surface rms accuracy is $0.35 \mathrm{~mm}$. Therefore, the antenna is suitable for observations at frequencies as high as $43 \mathrm{GHz}$. It is equipped with a digital base-band converter (DBBC) developed by the Italian Institute of Radio Astronomy, a Symmetricom Active Hydrogen Maser MHM-2010 (75001-114) and with the Mark5B + data recorder developed at MIT Haystack Observatory. However these were not installed at the time of this experiment, so data were recorded with the same equipment as installed at ASKAP-29.

The antenna elevation axis is at a height of approximately $7.1 \mathrm{~m}$ above the ground level. The elevation axis is supported by a pedestal of steel construction. The pedestal is essentially a steel cylinder of $\sim 2.5 \mathrm{~m}$ diameter. Apart from the pedestal all other components of the antenna (the 
Table 1. Specifications of an ASKAP antenna

\begin{tabular}{ll}
\hline Antenna type & Fully-steerable, prime focus \\
Reflector & 12 -metre paraboloid \\
Surface accuracy & $0.5 \mathrm{~mm}$ rms or better \\
Mount & 3 axes: polarization, elevation, azimuth \\
Height of elev. axis & $7.51 \mathrm{~m}$ above concrete foundation \\
Azimuth axis range & $-180^{\circ}$ to $+360^{\circ}$ \\
Azimuth axis speed & $3 \% \mathrm{~s}$ \\
Azimuth axis accel. & $3 \% \mathrm{~s}^{2}$ \\
Elevation axis range & $15^{\circ}$ to $89^{\circ}$ \\
Elevation axis speed & $1 \% \mathrm{~s}$ \\
Elevation axis accel. & $1 \% \mathrm{~s}^{2}$ \\
Polarization axis range & $-180^{\circ}$ to $+180^{\circ}$ \\
Polarization axis speed & $3 \% \mathrm{~s}$ \\
Polarization axis accel. & $3 \% \mathrm{~s}^{2}$ \\
\hline
\end{tabular}

Table 2. Specifications of a WARKI $2 \mathrm{M}$ antenna

\begin{tabular}{ll}
\hline Antenna type & Fully-steerable, dual-shaped Cassegrain \\
Manufacturer & Cobham/Patriot, USA \\
Main dish diam. & $12.1 \mathrm{~m}$ \\
Secondary refl. diam. & $1.8 \mathrm{~m}$ \\
Focal length & $4.538 \mathrm{~m}$ \\
Surface accuracy & $0.35 \mathrm{~mm}$ \\
Pointing accuracy & $18^{\prime \prime}$ \\
Frequency range & $1.4-43 \mathrm{GHz}$ \\
Mount & alt-azimuth \\
Azimuth axis range & $90^{\circ} \pm 270^{\circ}$ \\
Elevation axis range & $4.5^{\circ}$ to $88^{\circ}$ \\
Azimuth axis max speed & $5^{\circ} / \mathrm{s}$ \\
Elevation axis max speed & $1^{\circ} / \mathrm{s}$ \\
Main dish f/D ratio & 0.375
\end{tabular}

reflector and feed support structure) are constructed of aluminum. The support foundation for the antenna is a reinforced concrete pad that is $1.22 \mathrm{~m}$ thick by $6.7 \times 6.7$ meters square. The ground that the foundation is laid on consists of weathered sandstone/mudstone, i.e. it is of sedimentary origin, laid down in the Miocene period some 20 million years ago.

The radio telescope is directly connected to the regional advanced network KAREN (Kiwi Advanced Research and Education Network), which provides fast connectivity between New Zealand's educational and research institutions (Weston, Natusch \& Gulyaev 2010). At the time of the experiment, the Trans-Tasman (New ZealandAustralia) connectivity from the observatory was $155 \mathrm{Mbps}$. By the end of 2010 this was upgraded to 1 Gbps.

A preliminary survey has been conducted in collaboration with the New Zealand Crown Research Institute (CRI), GNS Science and Land Information New Zealand (LINZ) to determine an initial estimate of the reference point of the VLBI site WARK I 2M. This reference point is defined as the intersection of the azimuth and elevation axes of the telescope. A real-time kinematic (RTK) GPS method was used to derive the position with respect to the GPS station WARK.
Table 3. The network stations

\begin{tabular}{lccc}
\hline Name & $\phi_{g d}$ & $\lambda$ & Diam. \\
\hline ASKAP-29 & $-26^{\circ} .69$ & $116^{\circ} .64$ & $12 \mathrm{~m}$ \\
ATCAPN5 & $-30^{\circ} .31$ & $149^{\circ} .56$ & $22 \mathrm{~m}$ \\
HOBART26 & $-42^{\circ} .81$ & $147^{\circ} .44$ & $26 \mathrm{~m}$ \\
MOPRA & $-31^{\circ} .27$ & $149^{\circ} .10$ & $22 \mathrm{~m}$ \\
PARKES & $-33^{\circ} .00$ & $148^{\circ} .26$ & $64 \mathrm{~m}$ \\
WARKI 2M & $-36^{\circ} .43$ & $174^{\circ} .66$ & $12 \mathrm{~m}$ \\
\hline
\end{tabular}

WARK was established in November 2008 at the radio telescope site and is one of thirty-nine PositioNZ network stations (Blick \& Collett 2010) in New Zealand. ${ }^{5}$ All data received from the PositioNZ stations are compiled into 24-hour sessions and are processed to produce daily positions for each station in terms of ITRF2000. ${ }^{6}$ The coordinates for WARK used in the following calculations were derived by averaging the daily coordinate solutions for 2010 February 19 through March 09.

The RTK reference receiver was set up in an arbitrary location with clear sky view and was configured to record raw observations in addition to transmitting real-time corrections. This station was later post-processed with respect to WARK and all RTK rover-surveyed positions were subsequently adjusted relative to the updated reference position.

Several points on the rim of the main reflector were identified and each point was measured several times with the RTK rover while the telescope was repositioned in elevation and azimuth between successive measurements. The rover GPS antenna was mounted on a $0.5-\mathrm{m}$ survey pole and was held by hand for each measurement. Access to the rim of the reflector was achieved with a hydraulic cherry picker.

The sequence of observation for determination of the horizontal axis was as follows. The telescope azimuth axis was held fixed (nominally $0^{\circ}$ ). A point near the highest edge of the reflector was identified and measured with the telescope in four positions of elevation, from almost zenith $\left(\sim 88^{\circ}\right)$ to as high as the cherry picker could reach $\left(\sim 38^{\circ}\right)$. This was repeated a second time with a point identified on the edge of the reflector to one side of the telescope. Five positions of elevation were measured at this point, from $10^{\circ}$ to $80^{\circ}$.

The sequence of observation for determination of the vertical axis was as follows. The telescope elevation axis was held fixed (nominally $80^{\circ}$ ). Three points around the edge of the reflector were identified. The telescope azimuth axis was rotated into three positions such that each identified point could be measured consecutively from the surveyor's location in the cherry picker. The cherry picker was then repositioned twice around the perimeter of the telescope and the measurements were repeated at each

\footnotetext{
${ }^{5}$ http: / / www . linz.govt.nz/geodetic/positionz/.

${ }^{6}$ For more details see http://geonet.org.nz/resources/ gps / gps-processing-notes.html.
} 
Table 4. Coordinates of ASKAP-29 and WARKI $2 \mathrm{M}$ derived from analysis of experiment vt14a

\begin{tabular}{lcrc}
\hline Station & \multicolumn{3}{c}{ Station coordinates $(\mathrm{m})$} \\
& $X$ & $Y$ & $Z$ \\
\hline ASKAP-29 & $-2556741.89 \pm 0.09$ & $5097441.23 \pm 0.14$ & $-2847748.34 \pm 0.11$ \\
WARK I 2M & $-5115325.55 \pm 0.10$ & $477842.95 \pm 0.05$ & $-3767194.41 \pm 0.09$ \\
\hline
\end{tabular}

cherry picker location. This provided three measurements of each identified point with a fixed telescope zenith and varying azimuths.

The resulting points from these measurements describe two vertical circles of rotation which define the movable elevation axis and three horizontal circles of rotation which define the fixed azimuthal axis. The coordinates for all subsequent calculations were retained as geocentric Cartesian coordinates to avoid any possibility of errors related to transformation of projection.

The following method was used to determine the axes and their intersection point from these observations. The equation of a circle from three points (P. Bourke, 1990, internal $\mathrm{memo}^{7}$ ) was used to calculate all possible combinations of three observed points which define a circle of rotation. A simple mean was taken for all horizontal axis definitions and all vertical axis definitions. The midpoint of the closest point of approach of each axis to the other was used as the final estimate of the point of intersection. The distance between the closest point of approach on each axis was calculated to be $24 \mathrm{~mm}$. Based on the variation of results for different combinations of survey points, we estimate that the accuracy of the determined intersection point is within $0.1 \mathrm{~m}$.

In summary, the following coordinates of the intersection of the azimuth and elevation axes for the radio telescope WARK I $2 \mathrm{M}$ were derived in terms of ITRF2000 at the epoch of the survey (March 2010):

$$
\begin{aligned}
& X=-5115324.5 \pm 0.1 \mathrm{~m} \\
& Y=477843.3 \pm 0.1 \mathrm{~m} \\
& Z=-3767193.0 \pm 0.1 \mathrm{~m}
\end{aligned}
$$

It is intended that the radio telescope reference point coordinates will subsequently be re-determined to a higher accuracy with the use of a variety of terrestrial and GNSS survey techniques Dawson, Johnston \& Twilley (2004) and a more rigorous least-squares analysis of the observations. Four geodetic survey monuments have been built within $15-20 \mathrm{~m}$ of the antenna pedestal for this purpose.

\section{Geodetic VLBI Experiment}

Seven stations participated in the 7-hour VLBI experiment vt14a on 2010 May 07, with the goal of determining station coordinates. The stations are listed in Table 3 and

\footnotetext{
${ }^{7}$ Available at http://Iocal.wasp. uwa. edu.au/ pbourke/ geometry/circlefrom3.
}

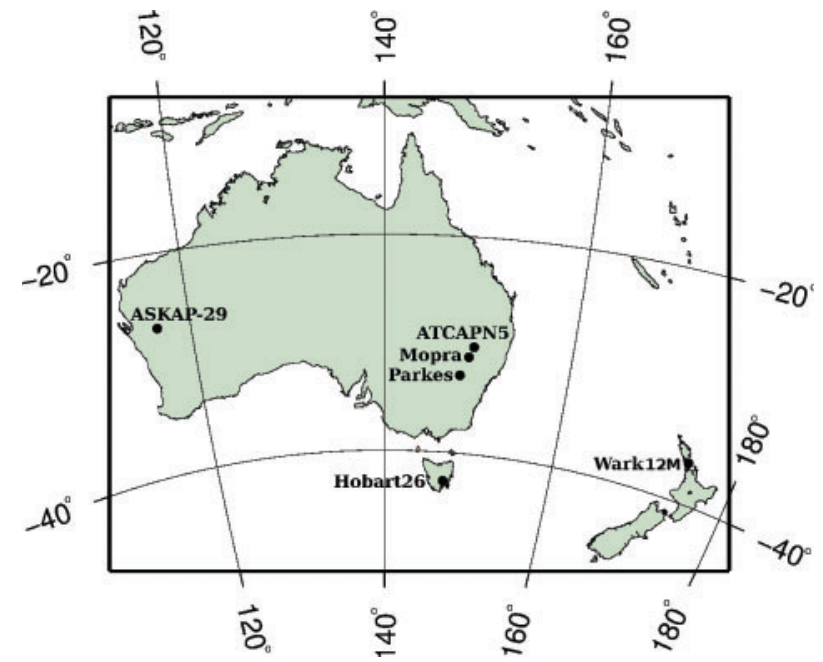

Figure 3 The LBA network used in vt14a experiment.

shown in Figure 3. Usually geodetic experiments are made in two bands recorded simultaneously at $2.2-2.3 \mathrm{GHz}$ and $8.0-8.8 \mathrm{GHz}$. Since group-delay errors are reciprocal to the frequency range, a wide range, $720 \mathrm{MHz}$, is used in VLBI experiments dedicated to geodesy. Radio waves are delayed in the ionosphere, and the magnitude of this delay depends on total electron content along the paths - a highly variable quantity. Since the contribution of the ionosphere to path delay is reciprocal to the square of effective frequency, simultaneous observations at two widely separated frequencies allow the formation of ionosphere-free combinations of group delays.

However, for this experiment the data were recorded in the band $1368-1432 \mathrm{MHz}$ in dual polarisation (i.e. a single $64 \mathrm{MHz}$ channel). Observations within a narrow band at a low frequency are highly unusual for geodeticstyle experiments since they are not able to provide accuracy comparable with dual-frequency, wide-frequency range experiments. However, we ran this experiment because in May 2010 the new antennas had only L-band receivers, and this experiment provided an opportunity not only to make a coarse estimate of station coordinates, but also to test the equipment. The single-frequency setup is a limitation of the VLBI backend available at both ASKAP-29 and WARKI $2 \mathrm{M}$ at the time of observations.

The experiment was scheduled using sur_sked software in the VLBI geodetic mode. A pool of 113 sources with correlated flux densities at X-band greater than 700 mJy based on results of the ongoing LBA Calibrator Survey (Petrov et al. 2011a) observing campaign was 
used. The scheduling goal was to have a uniform sky coverage at each station. This was achieved by putting a next source in the schedule that has a maximum score among other candidate sources. The score was assigned according to

$$
S=\frac{1}{t_{s}}\left(\frac{D_{\min }+0.1}{0.66}\right)^{4}
$$

where $t_{s}$ is maximum slewing time for all participating antennas in seconds, and $D_{\min }$ is the distance in radians from any source observed in the past. If a source was observed during the previous 150 minutes, it was barred from consideration as a candidate. Each scan had a nominal duration of $150 \mathrm{~s}$. Every hour a set of four atmosphere calibration scans was observed: two scans with all antennas with their elevation in the range $\left[12^{\circ}, 45^{\circ}\right]$, one scan at elevations $\left[32^{\circ}, 45^{\circ}\right]$ and one scan at elevations $\left[45^{\circ}, 90^{\circ}\right]$. The scheduling algorithm for each set found all combinations of calibrator sources that fell in the elevation ranges and selected the sequence of four objects that minimized the slewing time. The purpose of these calibration observations was to serve as amplitude and bandpass calibrators and to improve the robustness of estimates of the path delay in the neutral atmosphere.

\section{Data Analysis}

\subsection{Data Transfer and Correlation}

Following the observations, the data from ATCAPN5, HOBART26, MOPRA and PARKES were transferred via fast network connections to a petabyte data store operated by iVEC in Western Australia, with the support of ARCS (the Australian Research Collaboration Service). The data from WARK I $2 \mathrm{M}$ were transferred from the telescope using the trans-Tasman link directly to Perth. The data from ASKAP-29 were transported to Perth by car, as the fast network to the MRO at the time of the experiment was under construction. All data were later transferred to disks mounted on the correlation cluster at Curtin University and correlated using the DiFX software correlator (Deller et al. 2007). Initial antenna positions for WARKI $2 \mathrm{M}$ and ASKAP-29 determined from GPS receivers were used for correlation. Other station positions are known from VLBI solutions with accuracy better than one centimeter (Petrov et al. 2009a). The data were correlated using an integration period of $0.25 \mathrm{~s}$ and 512 frequency channels across the 64-MHz band. All four combinations of right and left circular polarization signals were correlated.

\subsection{Data Quality Control Made at the Correlator}

Initial data-quality checking and clock searching was performed at the correlator using an automated pipeline script implemented using the ParselTongue AIPS interface (Kettenis et al. 2006). Hydrogen masers were used to provide the frequency standard at all stations except at ASKAP-29 where a lower-stability (but still acceptable) rubidium frequency standard was used. This resulted in a noticeable correlation loss on baselines to ASKAP-29. All stations except WARK I 2M observed with dual circular polarizations, while WARKI2M observed dual linear polarizations due to a malfunction of the linear-to-circular conversion module, resulting in a reduced signal-to-noise ratio on WARKI2M baselines: $\sim \sqrt{2}$ less sensitive than expected from a priori SEFD estimates. ATCA participated as a phased array comprising $5 \times 22$-m dishes. Polarization isolation at the PARKES receiver did not work correctly during the first two hours of the experiment, but this was fixed after the linear-to-circular hybrid module was reset.

\subsection{Post-Correlator Data Analysis}

The spectrum of cross-correlation and autocorrelation for each scan and each baseline computed by the correlator at a uniform two-dimensional grid of accumulation periods and frequencies was used for further processing.

The fringe-fitting procedure searches for phase delay $\tau_{p}$, phase delay rate $\dot{\tau}_{p}$, group delay $\tau_{g}$, and its time derivative $\dot{\tau}_{g}$ that correct their a priori values used by the correlator model in such a way that the coherent sum of weighted complex cross-correlation samples over a given baseline and a given scan, $c_{i j}$,

$$
\begin{aligned}
& C\left(\tau_{p}, \tau_{g}, \dot{\tau}_{p}, \dot{\tau}_{g}\right)=\sum_{i} \sum_{j} c_{i j} w_{i j} \\
& \quad \times e^{i\left(\omega_{0} \tau_{p}+\omega_{0} \dot{\tau}_{p}\left(t_{i}-t_{0}\right)+\left(\omega_{j}-\omega_{0}\right) \tau_{g}+\left(\omega_{j}-\omega_{0}\right) \dot{\tau}_{g}\left(t_{i}-t_{0}\right)\right)}
\end{aligned}
$$

reaches the maximum amplitude. Index $i$ runs over time and index $j$ runs over frequencies. $\omega_{0}$ and $t_{0}$ denote the angular reference frequency within the band and the reference time within a scan and $w_{i j}$ are weights that are defined as a fraction of processed samples in each accumulation period. The software PIMA was used for amplitude calibration and evaluation of phase and group delay, as well as their time derivatives. The algorithm implemented in PIMA is described in detail in Petrov et al. (2011b).

After computing phase and group delays and their derivatives, the spectrum of cross-correlations can be averaged over time or frequency. This provides valuable diagnostics. The amplitude spectrum at the new stations ASKAP-29 and WARKI2M shows a significant drop of the amplitude near the edges of the band as it is seen in Figure 4. The signal-to-noise ratio losses due to the shape of the filter are at a level of $13 \%$. A portion of the band with the fringe amplitude less than one quarter of the maximum was filtered out in the final processing.

Since the observations were made during low solar activity, the ionosphere did not cause significant decorrelation. Instability of the rubidium frequency standard is clearly seen in Figure 5, and it caused a decorrelation on baselines with ASKAP-29 at a level of 4-5\%.

At the first step of geodetic data analysis, the spectrum of the cross-correlation function was screened. The edges of the bandpass and several spurious peaks near the center of the bandpass were filtered out. The complex bandpasses were evaluated and the fringe-fitting 

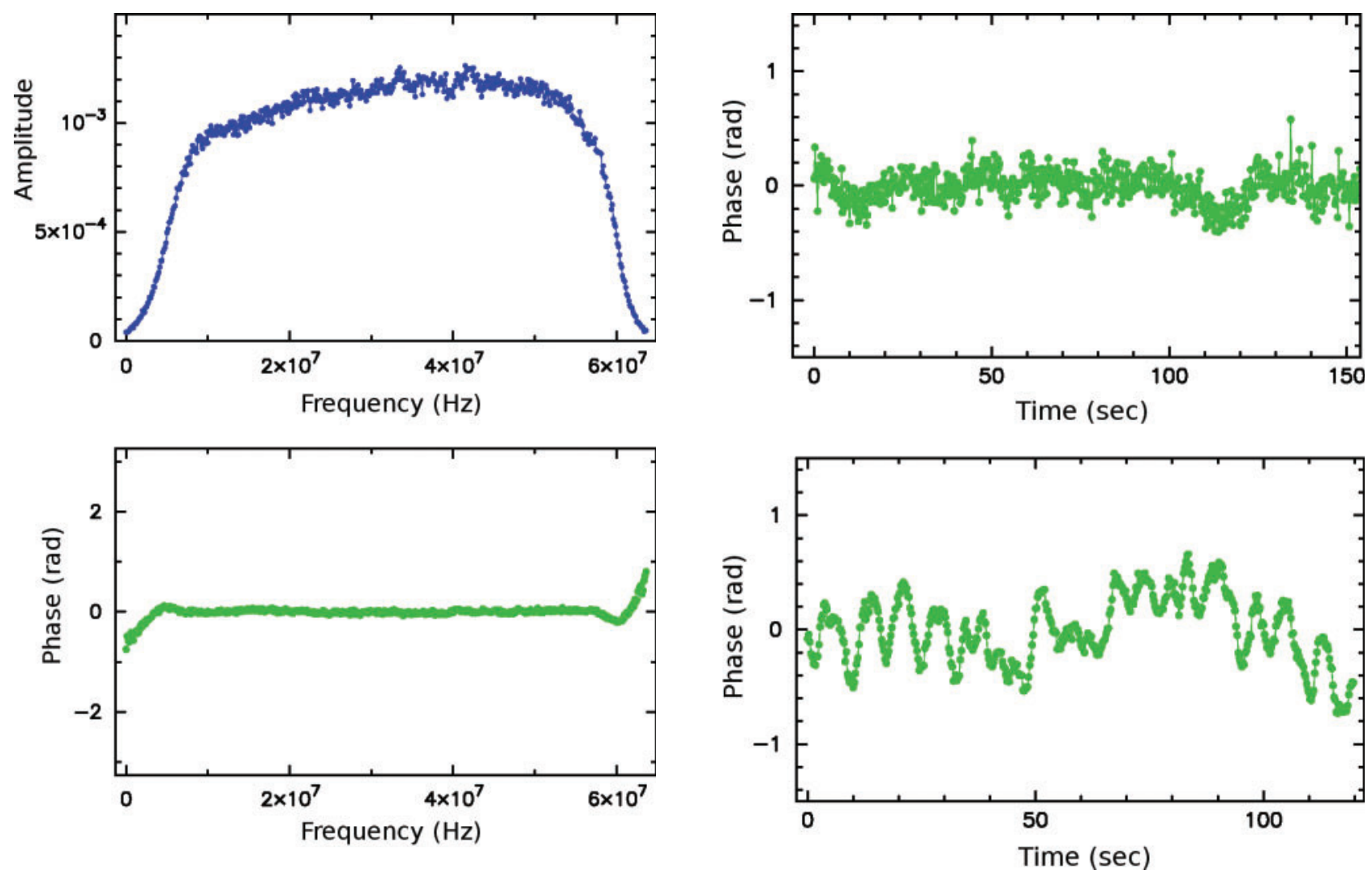

Figure 4 The amplitude (upper plot) and phase (lower plot) of the cross-correlation spectrum in a scan of source 0537-441 at baseline ASKAP-29/PARKES averaged over time after applying fringe search. Integration time $0.25 \mathrm{~s}$.

procedure was repeated with the the refined bandpasses and with the bandpass mask applied. This procedure is described in full detail in Petrov et al. (2011b). Analysis of the amplitudes at all combinations of right and left circular polarizations, RR, LL, LR and RL, confirmed that the polarization setup at PARKES for the first two hours was incorrect. But it was also found that polarization impurity, defined as $\sqrt{|R L|^{2}+|L R|^{2}} / \sqrt{|R R|^{2}+|L L|^{2}}$, was significant on baselines with other stations. For example, the polarization impurity at baseline ATCAPN5/MOPRA was at a level 7\%, on baselines ATCAPN5/HOBART26, ATCAPN5/ ASKAP-29 and ATCAPN5/PARKES Was 25-30\%, and the baseline ATCAPN5/WARKI2M was $\sim 100 \%$. Therefore, the coherent combining dual polarization improved the signal-to-noise ratio at some baselines and degraded it at others. For this reason, the datasets of group delays with $\mathrm{RR}$ and LL polarizations were used as independent experiments in further processing.

The next step of the analysis pipeline is to compute theoretical path delays and form small differences between them and derived group delays. Computation of theoretical path delays was made with the use of the VTD software $^{8}$ that incorporates state-of-the art geophysical models. Our computation of theoretical time delays in general follows the approach of Sovers, Fanselow \&

\footnotetext{
${ }^{8}$ http: / /astrogeo. org/vtd.
}

Figure 5 The phase of the cross-correlation spectrum in a scan of source 0537-441 at baseline WARKI2M/PARKES (upper plot) and ASKAP-29/PARKES (lower plot) averaged over frequency after applying fringe search solutions.

Jacobs (1998) with some refinements. The most significant ones are the following. The advanced expression for time delay derived by Kopeikin \& Schäfer (1999) in the framework of general relativity was used. The displacements caused by the Earth's tides were computed using the numerical values of the generalized Love numbers presented by Mathews (2001) following a rigorous algorithm described by Petrov \& Ma (2003) with a truncation at a level of $0.05 \mathrm{~mm}$. The displacements caused by ocean loading were computed by convolving the Green's functions with ocean tide models. The GOT99.2 model of diurnal and semi-diurnal ocean tides (Ray 1999), the NAO99 model (Matsumoto, Takanezawa \& Ooe 2000) of ocean zonal tides, the equilibrium model (Petrov \& Ma 2003) of the pole tide, and the tide with period of 18.6 years were used. Station displacements caused by the atmospheric pressure loading were computed by convolving the Green's functions that describe the elastic properties of the Earth (Farrell 1972) with the output of the atmosphere NCEP Reanalysis numerical model (Kalnay et al. 1996). The algorithm of computations is described in full detail in Petrov \& Boy (2004). The displacements due to loading caused by variations in soil moisture and snow cover in accordance with the GLDAS Noah model (Rodell 2004) with a resolution $0.25^{\circ} \times 0.25^{\circ}$ were computed using the same technique as the atmospheric pressure loading. The empirical model of harmonic variations 
in the Earth orientation parameters heo_20101111 derived from VLBI observations according to the method proposed by Petrov (2007) was used. The time series of UT1 and polar motion derived by the NASA Goddard Space Flight Center operational VLBI solutions were used a priori.

The a priori path delays in the neutral atmosphere in the direction of observed sources were computed by numerical integration of differential equations of wave propagation through the heterogeneous media. The fourdimensional field of the refractivity index distribution was computed using the atmospheric pressure, air temperature and specific humidity taken from the output of the Modern Era Retrospective-analysis for Research and Applications (MERRA) (Schubert et al. 2008). That model presents the atmospheric parameters on a grid $1 / 2^{\circ} \times 2 / 3^{\circ} \times 6^{\mathrm{h}}$ at 72 pressure levels.

In the initial least square (LSQ) solution, positions of all stations except PARKES were estimated, as well as coefficients of the expansion of clock function and the residual atmosphere path delay in the zenith direction into the B-spline basis of the first degree. The quality check revealed a clock break at station HOBART26. During the preliminary phase of the data analysis, outliers were eliminated and the baseline-dependent corrections to the a priori weights, defined to be reciprocal to formal uncertainties of group delays, were determined in such a way that the ratio of the weighted sum of squares of residuals to their mathematical expectation was close to unity. Group delays from RR and LL polarization data were processed independently.

The final LSQ solution used all VLBI group delays collected from 1984 through $2010,{ }^{9}$ a total of 7.5 million values, including 824 group delays from this experiment. Positions of all stations, all sources, the Earth orientation parameters and over 1 million nuisance parameters were estimated in a single LSQ run. Minimal constraints were imposed to require that net translation and net rotation over new position estimates of 48 stations with long histories with respect to positions of observations to these stations in the ITRF2000 catalogue (Altamimi, Sillard \& Boucher 2002) to be zero. This ensures that positions of all stations, including ASKAP-29 and WARK I 2M, are consistent with the ITRF2000 catalogue. More details about theparameter estimation technique can be found in Petrov et al. (2009b).

The estimates of coordinates of ASKAP-29 and WARK I $2 \mathrm{M}$ on epoch 2010.05.07 are given in table 4. The errors reported in the table are the formal uncertainties from the LSQ solution computed in accordance with the error propagation law. Since there was only one experiment available with rather an unusual setup, it is difficult to provide a realistic estimate of errors.

The largest source of systematic error is the path delay in the ionosphere. Our attempt to use maps of the total

\footnotetext{
${ }^{9}$ Available at the IVS Data Center at http: / / ivscC.gsfc.nasa . gov/products-data/index.html.
}

electron content (TEC) from GPS using the data product from the analysis center CODE did not improve the fit and shifted estimates of station coordinates at a fraction of the formal uncertainty $(0.2 \sigma)$. Analysis of dual-band VLBI experiments showed that global TEC maps above Australia and New Zealand provided by the International Global Navigation Satellite System Service are not reliable, and the path delay computed from this model poorly represents the true delay in the ionosphere, at least during solar minimum (Petrov et al. 2011a).

The adjustments to the residual zenith path delay in the atmosphere were in the range of $1-5 \mathrm{~ns}$, while they are typically in the range $0.03-0.1 \mathrm{~ns}$ for dual-band observations. Unlike the estimation of the mismodeled troposphere path delay, estimation of unmodeled ionosphere path delay in the zenith direction from the observations themselves does not adequately represent the true path delay, since when antennas point in different directions, the ionosphere-piercing points with a typical height of $\sim 450 \mathrm{~km}$ may be located at distances of up to $1000 \mathrm{~km}$.

For evaluation of the robustness of our coordinate estimates we made a trial solution and estimated the position of station ATCAPN5 independently. Estimates of the position of ATCAPN 5 appeared within $2 \sigma$ of the CATN5 pad. The position of CATN5 was derived from the position of the pad CATW104 determined from previous VLBI observations and from the results of a local survey that measured coordinates of CATN5 with respect of CATW104 (Petrov et al. 2009a). This gave us a hint that the reported uncertainty may be underestimated by a factor of 2, especially for the vertical component. In our final solution we applied the eccentricity vector between CATN5 and CATW104 from the local survey.

The data from RR and LL polarizations have independent random errors, but they share the same systematic errors. In another trial solution we processed RR and LL data separately. The position differences of these two solutions were within $1 \sigma$ for the horizontal component and $2 \sigma$, or $0.4 \mathrm{~m}$, for the vertical component.

However, the latter test is not sensitive to common systematic errors caused by unmodeled ionosphere path delays. In order to evaluate the magnitude of the ionosphere path delay errors, we used prior observations at the baseline PARKES/HOBART26 under the IVS geodetic program. We selected twenty-one 24-hour experiments in 2005-2010, the period of solar minimum. We ran three sets of 21 trial solutions. Within each set we used all experiments from 1980 through 2010 with station HOBART 26 removed from all but one experiment. The only experiment with HOBART 26 was different in each solution within a set. In such an experiment all other stations except HOBART 26 and PARKES were removed. This setup emulated the case of determining station coordinates from one experiment only. The reference set $A$ used an ionosphere-free combination of X- and S-band group delays. The set $B$ used X-band group delays for an experiment with HOBART2 6 and X/S combinations for all other experiments. The set $C$ used S-band group delays. We obtained 


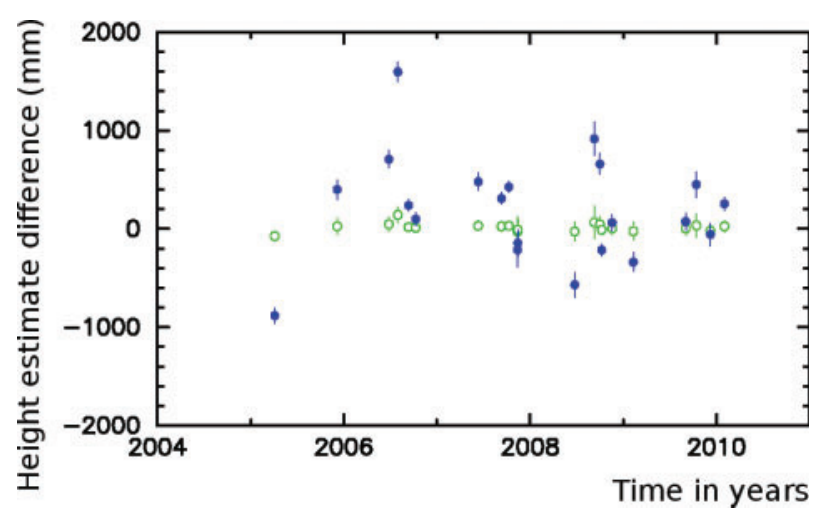

Figure 6 The differences in the vertical coordinate estimate of station HOBART26 from a series of trial solutions, when position was estimated only from one experiment at a single baseline with PARKES. Hollow circles represent the difference between the X-band-only solution and the X/S dual-band solution. The weighted root mean square (wrms) is $40 \mathrm{~mm}$. Filled discs represent the difference between the S-band-only solution and the X/S dual-band solution. The wrms is $490 \mathrm{~mm}$.

three series of 21 estimates of HOBART26 positions. We formed the difference between estimates of HOBART26s vertical and horizontal coordinates between sets $B$ and $A$, and between sets $C$ and $A$. The differences for the vertical component of site position estimates are given in Figure 6.

The only difference between the solutions in sets $A$, $B$, and $C$ is the treatment of the ionosphere path delay. Therefore, we can consider the differences $B-A$ and $C-A$ to be primarily due to the contribution of unmodeled ionosphere path delay to estimates of site position. The average ratio of differences $(B-A) /(C-A)$ is 13.7 which is very close to the square of the ratio of effective ionosphere frequencies at $\mathrm{X}$ and $\mathrm{S}$ bands: $(8.387 / 2.250)^{2} \approx$ 13.9. This supports our argument that the differences $C-A$ are due to the ionosphere path delay at $\mathrm{S}$ band. Since the differences $B-A$ and $C-A$ are nicely scaled with the square of effective frequency, we can extrapolate the estimates of the weighted root mean square (wrms) of site-position time series from solution $C$ to the $\mathrm{L}$ band by scaling them by $(2.250 / 1.4)^{2} \approx 2.6$. If we were determining the position of HOBART 26 from one of these twenty-four experiments at $\mathrm{L}$ band, the wrms of position errors due to unaccounted ionosphere path delay would have been $1.3 \mathrm{~m}$ for the vertical component and $0.14 \mathrm{~m}$ for the horizontal component. We extrapolate these numbers to our estimates of ASKAP-29 and WARKI $2 \mathrm{M}$ positions.

The differences in position estimates of WARK I $2 \mathrm{M}$ from VLBI and from GPS surveys, VLBI - GPS, are $1.66 \mathrm{~m}$ for the vertical component, $0.49 \mathrm{~m}$ for the east component, and $-0.48 \mathrm{~m}$ for the north component. The difference in the vertical component can be explained by the effect of the ionosphere.

\section{Summary and Future Work}

We obtained the first estimates of the positions of ASKAP-29 and WARKI2M antenna reference points from
VLBI observations. The random position $1 \sigma$ errors, 5$6 \mathrm{~cm}$ for the horizontal coordinates and $20-40 \mathrm{~cm}$ for the vertical component, are close to that what one can expect from narrow-band observations at $\mathrm{L}$ band. It is a pleasant surprise that the very first observations that followed the successful fringe test yielded a reasonable result. However, the systematic errors due to unaccounted ionosphere are significantly greater: $1.3 \mathrm{~m}$ for the vertical component and $0.14 \mathrm{~m}$ for the horizontal component. We also identified several problems with station equipment that will be fixed in the future. The use of a rubidium frequency standard at ASKAP-29 caused a decorrelation at a level of 4-5\% within 2-minute long scans. The frequency instability at longer time intervals appeared negligible with respect to errors caused by the ionosphere.

The existing L-band (1.4-GHz) receiver on ASKAP-29 will be replaced in early 2011 with a new receiver comprising a purpose-built feed horn optimized for this antenna, with significantly higher efficiency, and a noise calibration source. In 2011 ASKAP-29 may be equipped with an X-band (8.4-GHz) receiver, and the possibility of an upgrade to a higher frequency receiver is being considered.

In 2011, WARK I 2M was equipped with $\mathrm{S} / \mathrm{X}$ dual-band receivers. Future geodetic experiments at these frequencies are planned for February 2011. The participation of stations ASKAP-29 and WARKI2M in VLBI experiments under absolute astrometry and geodesy programs will permit an improvement in the precision of results by two orders of magnitude and reach a millimeter level of accuracy. A more precise survey to provide $1-3 \mathrm{~mm}$ accuracy of the tie vector between VLBI and GPS antenna reference points is planned. By combining this with further VLBI observations it will be possible to reconcile the currently observed differences between GPS and VLBI positions of the WARKI $2 \mathrm{M}$ antenna reference point.

In November 2010, Telecom New Zealand made its 30-metre satellite Earth station available to AUT's Institute for Radio Astronomy and Space Research. It is a wheel-and-track beam-waveguide antenna built by NEC Corporation in 1984. It will be converted to a radio telescope capable of conducting both astronomical and geodetic research.

\section{Acknowledgments}

This work uses data obtained from the MRO, jointly funded by the Commonwealth Government of Australia and the Western Australian State government. The MRO is managed by the CSIRO. We acknowledge the Wajarri Yamatji people as the traditional owners of the Observatory site. The Long Baseline Array is part of the Australia Telescope National Facility which is funded by the Commonwealth of Australia for operation as a National Facility managed by CSIRO. The International Centre for Radio Astronomy Research is a Joint Venture of Curtin University and The University of Western Australia, funded by the Western Australian State government. 
iVEC is a joint venture between CSIRO, Curtin University, Edith Cowan University, The University of Western Australia and Murdoch University and is supported by the Western Australian Government. SJT is a Western Australian Premier's Research Fellow, funded by the Western Australian government. This project was supported in part by the Australian Research Collaboration Service (ARCS). We used in our work the dataset MAI6NPANA provided by the NASA/Global Modeling and Assimilation Office (GMAO) in the framework of the MERRA atmospheric reanalysis project.

\section{References}

Alexander, F. E. S., 1946, Radio \& Electronics, 1(1), 16-17, 20 Altamimi, Z., Sillard, P. \& Boucher, C., 2002, JGR, 107, 2214 Blick, G. \& Collett, D., 2010, Survey Quarterly, 63, 20

Bolton, J. G., Stanley, G. J. \& Slee, O. B., 1949, Natur, 164(4159), 101

Bolton, J. G., 1982, in Proceed. Astronom. Soc. Austr. 4(4), 349

Charlot, P. et al., 2002, in the 6th EVLBI Network Symposium, 9 http://mpifr-bonn.mpg.de/div/vlbi/evn2002/ bookPCharlot.pdf

Collett, D., 2010, in Proceeding of FIG Congress 'Developing a National Real-time CORS Network in New Zealand' http: / / www. linz.govt.nz/geodetic/standards-publi cations/published-paper-presentations / col lett-national-real-time-cors-network.pdf

Dawson, J., Johnston, G. \& Twilley, B., 2004, in Proceedings of the IERS Workshop in site co-location, IERS Technical Note, No 33. 128

Deller, A. T., Tingay, S. J., Bailes, M. \& West, C., 2007, pasp, 119, 318

Farrell, W. E., 1972, Rev. Geophys. Spac. Phys., 10(3), 751

Gulyaev, S., Natusch, T., Addis, B., Tingay, S. \& Deller, A., 2005, Southern Stars, 44(4), 12

Gulyaev, S. \& Natusch,T., 2009, In International VLBI Service for Geodesy and Astrometry 2008 Annual Report, NASA/TP-2009214183, Eds. Behrend, D. \& Baver, K. D., 68 ftp : / / ivscc . gsfc.nasa.gov/pub/annual-report/2009/pdf/ nswark.pdf
Johnston, S. et al., 2008, ExA, 22(3), 151

Kalnay, E. M. et al., 1996, Bull. Amer. Meteorol. Soc., 77, 437-471

Kettenis, M., van Langevelde, H. J., Reynolds, C. \& Cotton, B., 2006, Astron. Data Anal. Software Syst. XV, 351, 497

Kopeikin, S. M. \& Schäfer, G., 1999, Phys Rev D, 60(12), 124002

Mathews, P. M., 2001, J Geod Soc Japan, 47(1), 231

Matsumoto, K., Takanezawa, T. \& Ooe, M., 2000, J Oceanography, 56,567

Petrov, L., 2007, A\&A, 467(1), 359

Petrov, L. \& Ma, C., 2003, JGR, 108(B4), 2190

Petrov, L. \& Boy, J.-P., 2004, JGR, 109, B03405

Petrov, L., Phillips, C., Bertarini, A., Deller, A., Pogrebenko, S. \& Mujunen, A., 2009a, PASA, 26, 75

Petrov, L., Gordon, D., Gipson, J., MacMillan, D., Ma, C., Fomalont, E., Walker, R. C. \& Carabajal, C., 2009b, J. Geodesy, 83(9), 859

Petrov, L., Phillips, C., Bertarini, A., Murphy T. \& Sadler E. M., 2011a, MNRAS, in press, preprint (astro-ph/1012.2607)

Petrov, L., Kovalev, Y. Y., Fomalont, E., Gordon, D., 2011b, AJ, submitted, preprint (astro-ph/1101.1460)

Ray, R. D., 1999, NASA/TM-1999-209478, Greenbelt, MD USA

Rodell, M. et al., 2004, Bull. Amer. Meteor. Soc., 85(3), 381

Sarti, P., Sillard, P. \& Vittuari, L., 2004, J Geodesy, 78(3), 210

Sarti, P., Abbondanza, C. \& Vittuari, L., 2009, J Geodesy, 83(11), 1115

Schubert S. et al., 2008, In Proc. of Third WCRP International Conference on Reanalysis, Tokyo, V1-104 (http: / / wcrp. ipsl.jussieu.fr/Workshops/Reanalysis2008/ Documents/V1-104 ea.pdf)

Smits, R., Tingay, S. J., Wex, N., Kramer, M. \& Stappers, B., 2010, A\&A, submitted

Sovers, O. J., Fanselow, J. L. \& Jacobs, C. S., 1998, Rev Modern Phys, 70, 1393

Tingay, S. J. et al., 2006, ATNF Newsletter, 58, 8-10 http: / / www. atnf.csiro.au/news/newsletter/feb06/ATNF news_06Feb.pdf

Tzioumis, A. K. et al., 2010, AJ, 140(5), 1506

Weston, S., Natusch, T., Gulyaev, S., 2010, in Procedings of the 17th Electronics New Zealand Conference, submitted (preprint astro$\mathrm{ph} / 1011.0227)$ 